\title{
Investing in primary care as the bridge for transitional care
}

\section{Finella Craig, ${ }^{1}$ C Dilini Rajapakse, ${ }^{1}$ Katrina McNamara, ${ }^{2}$ Neil Williamson ${ }^{3}$}

Transition to adult services of young people with palliative care needs is the crisis riding the wave of advances in paediatric medicine. The review by Doug et al has highlighted the extent of the crises we are facing, with a lack of standardised transition programmes, a poor evidence base to transition guidelines and insufficient outcome data to evaluate the different models. How, in palliative care, have we found ourselves in this position, with a long-anticipated patient population that has no clear destination and little means of getting there?

One of the difficulties must stem from the broad scope of paediatric palliative care, where early referral in the course of a life-threatening or life-limiting condition is the norm. ${ }^{2}$ Consequently, those transitioning to adult services encompass a diversity of diagnoses, disease groups and disease trajectories. One of the dilemmas facing paediatricians is not "should I transition my young adult patients" but "how and where do I transition them to". It is no surprise that published models of good practice, although limited, were mainly identified for condition-specific transition programmes, notably cystic fibrosis, cancer and congenital heart disease (CHD). Here, a single disease group, managed by disease-specific specialists in paediatrics, has a clearly identifiable disease-specific service in adult medicine. Of course, 'palliative care' also has an equivalent, clearly identified and developed specialty in adult services, so why is there so little evidence of transition programmes from paediatric to adult palliative care services?

Perhaps, as the authors highlight, the lack of evidence represents a shift in paediatric palliative care from terminal care to one that includes long-term

${ }^{1}$ Great Ormond Street Hospital for Children NHS Trust, London, UK

${ }^{2}$ ACT, Bristol, UK

${ }^{3}$ Richard House Children's Hospice, London, UK

\section{Correspondence to}

Dr Finella Craig, Great Ormond Street Hospital for Children NHS Trust, Great Ormond Street, London WC1N3JH, UK; craigf@gosh.nhs.uk supportive care. Consequently, young adult graduates from paediatric palliative care do not 'fit' into existing adult palliative care programmes, generally more focused on patients with advanced progressive illness. ${ }^{3}$ This 'poor fit', however, is not unique to palliative care. In 2003 the Task Force on the Management of Grown Up Congenital Heart Disease of the European Society of Cardiology identified that "the 'new population' of patients with CHD no longer fits within traditional divisions of training and practice" ${ }^{4}$ and emphasised the need to reintegrate paediatric and adult cardiac services in order to provide smooth transition.

\section{Editorial on the paper by Doug et al (see page 78)}

Doug et $a l^{1}$ were able to identify four transition models for CHD and while there remains controversy over the best model, the lack of evidence of similar planning between paediatric and adult palliative care services is disappointing.

Paediatricians, at least in part, must hold some accountability for the problem we now face. Several of the papers reviewed highlighted a reluctance to initiate transition owing to concerns that adult colleagues may not be familiar with the young person's palliative care needs or with paediatric diseases. However, holding 'back' young adults in paediatric services, for whatever reason, significantly undermines their role and value in adult society. Additionally, paediatricians may be unfamiliar with adult models of care, services and therapeutics, so may not be providing the best quality of care. As the authors have emphasised, providing services that respect both age and developmental stage is essential.

That studies were mainly limited to child health providers and settings perhaps reflects an additional problem in that adult providers have failed to sufficiently acknowledge the growing need for new or changing services. Despite evidence of transition planning in CHD, the 'blended model' of care was often used, with young people remaining with their paediatric cardiologist because adult congenital heart centres and adult cardiologists experienced in CHD were lacking. While the authors call for research originating from adult services, we believe that joint working and research between adult and paediatric services is essential. A key challenge for paediatricians is to lead in the education and training of adult providers and in identifying the types of services needed. Adult services must take the lead in commissioning and developing the services required to appropriately support this emerging adult population.

It was disappointing, but not surprising, to see that high quality parentprovider relationships were strongly associated with addressing transition issues. This suggests that young people without strong parent advocates may be receiving a poorer quality of support for transition. Although acknowledging that the role of the family is crucial, it is essential that transition programmes are developed around the needs of the client group and are not parent-dependent. Individualised transition plans seem to be key. Even where young people have the same underlying condition, their transition needs will vary depending on family dynamics that are intrinsic to paediatrics. A young man with Duchenne muscular dystrophy, for example, may be independently and emotionally a young adult in one family, but childlike and unwilling to be separated from his mother in another. How would you standardise place of care in hospital where admission to paediatric wards ends at 16 years and adult wards do not allow parents to sleep at the bedside?

Doug et al ${ }^{1}$ suggest a key worker should be involved in the transition process and we believe this could go some way to ensuring that transition is driven by the young person, alongside their parent. It is noted that peer-led transition models require further exploration, but this model may not be appropriate for young people with cognitive impairment or significant communication difficulties.

The lack of good quality transition data identified in this review highlights the urgent need for public health planners to collect the evidence and data needed to effectively plan the required service developments. While the focus of the study is predominantly healthcare, planning and evaluation of transition services must ensure common 
processes between the varieties of services these young people require: health, social care, education and employment. For young adults to remain in paediatric services, to continue living with parents because there is no acceptable funded alternative and to have no meaningful daytime activities is unacceptable. The use of validated outcome measures is crucial, but may prove challenging owing to a lack of standardised service provision in paediatric and adult services and across different geographical areas and disease groups. Patient and family feedback data were significantly lacking from publications, yet are essential to improve quality and to build on good practice models.
There is at present a growing trend to reintroduce the general practitioner (GP) during transition to provide the generalist overview and to be able to refer back to adult specialist services. Doug et $a l^{1}$ observe that this primary care transition model has met considerable opposition because of a lack of integration between primary and specialist care. Perhaps, as paediatricians, we should be working harder to support the role of the GP during childhood-the one service that provides lifelong continuity in healthcare, regardless of age or diagnosis.

Accepted 14 July 2010

Published Online First 18 September 2010

Arch Dis Child 2011:96:1-2.

doi:10.1136/adc.2010.191478
Competing interests None.

Provenance and peer review Commissioned; internally peer reviewed.

\section{REFERENCES}

1. Doug M, Adi Y, Williams J, et al. Transition to adult services for children and young people with palliative care needs: a systematic review. Arch Dis Child 2011;96:78-84.

2. ACT (Association for Children's Palliative Care). A Guide to the Development of Children's Palliative Care Services. 2009.

3. National Institute for Health and Clinical Excellence. Improving Supportive and Palliative Care for Adults with Cancer. 2004.

4. The Task Force on the Management of Grown Up Congenital Heart Disease of the European Society of Cardiology. Management of grown up congenital heart disease. Eur Heart J 2003;24:1035-84. 